\title{
An estimate of maintenance efficiency in Brown-Proschan imperfect repair model with bathtub failure intensity
}

\author{
Makram Krit ${ }^{1}$, Abdelwaheb Rebai ${ }^{2}$ \\ ${ }^{1}$ Institute Supérieur d'Administration des Entreprises, ${ }^{2}$ Ecole Supérieur de Commerce (TUNISIE) \\ kritmakram@yahoo.fr; abdelwaheb.rebai@fsegs.rnu.tn
}

Received January 2011

Accepted September 2011

\section{Abstract:}

Purpose: Estimate the maintenance efficiency in the Brown-Proschan model with the bathtub failure intensity.

Design/methodology/approach: Empirical research through which we propose a framework to establish the characteristics of failure process and its influence on maintenance process.

Findings: The main contribution of the present study is the reformulation of the Brown and Proschan model using the bathtub failure intensity

Practical implications: Our model is defined by BP reformulation one using bathtub failure intensity. This form of intensity is presented like superposition of two NHPP and Homogeneous Poisson one.

Originality/value: This is the follow on research on the study that employed the power-law-process type of failure intensity.

Keywords: repairable system, reliability, bathtub failure intensity, virtual age, imperfect maintenance, estimation, likelihood 


\section{Introduction}

The totality of the significant industrial systems is subjected to the actions corrective and preventive maintenance which are supposed to prolong their functional life. The efficiency evaluation of these maintenance actions is of a great practical interest, but it was seldom studied. In the literature, several models of maintenance effect were proposed. That is to say for example, Wang (2002) and Baxter, Kijima and Tortorella (1996). The authors tried to classify various models of maintenance. Particularly, a very significant characteristic to consider is the evaluation of the system failure intensity, and primarily the discovery at the appropriate time of its degradation. Moreover, to optimize the maintenance programs respecting the availability and to reduce the maintenance costs using the maintenance optimization by reliability (MOR), as it was the case in Jiang, Ji and Xia (2003) and Finkelstein (2008). More clearly, it is a question on the one hand of building stochastic models of failures process and repairs of various systems, and on the other hand, of implementing the statistical methods to exploit the failures and maintenances data raised by experts with an aim to evaluate the performance of these systems such as Doyen (2004).

The majority of these models consider only the corrective maintenance (CM) effect, known under the name of repair models. These models are useful to model the real systems which are supported by a constant repair. Several repair models, including those of Brown-Proschan, the Block, Borges and Savits model (1985), the Kijima model (1989), the most general models of Dorado, Hollander and Sethuraman (1997) and Last-Szekli model (1998), were all useful in this respect. Several theoretical properties, as well as the parameters estimators of fundamental failure intensity and their asymptotic intervals confidence studied by these authors, without evaluating the maintenance efficiency. The same claims of these models can be also used for the only preventive maintenance (PM).

The idea of the Brown-Proschan model (1983) is that, the efficiency of the $k^{\text {th }}$ maintenance action is evaluated by a random variable $E_{k}$, independently and identically distributed according to the Bernoulli law with parameter $p$, such as:

$$
E_{k}=\left\{\begin{array}{l}
1 \text { if the maintenance is perfect } \\
0 \text { if the maintenance is minimal }
\end{array}\right.
$$

Knowing that maintenance is always minimal over all the improvement period and that of service life (i.e. for $\mathcal{T}_{k} \leq \gamma_{1}$ ). We can show that at the time $t$ after $\gamma_{1}$, the 
duration passed since the last perfect maintenance (moreover, before the moment $\gamma_{1}$, all maintenances are supposed to be minimal) can be expressed in the form:

$$
t-\mathcal{T}_{\mathcal{N}_{t}}+\sum_{k=\mathcal{N}_{\gamma_{1}}+1}^{\mathcal{N}_{t}}\left[\Pi_{z=k}^{\mathcal{N}_{t}}\left(1-E_{z}\right)\right] x_{k}
$$

where $\mathcal{N}_{\gamma_{1}}=i+j$ represent the failures number (of maintenance action) will take place during, respectively the improvement and service life periods : i.e. before the instant $\gamma_{1}$. Under these conditions, the failure intensity is written by the sample principle given in the study of Dijoux (2009):

$$
\lambda_{t}(\mathcal{N}, E)=\left\{\begin{array}{lr}
\frac{1}{\eta_{0}}+\frac{\beta_{1}}{\eta_{1}^{\beta_{1}}}\left(t^{\beta_{1}-1}-\gamma_{0}^{\beta_{1}-1}\right) & \text { if } 0<t<\gamma_{0} \\
\frac{1}{\eta_{0}} & \text { if } \gamma_{0} \leq t \leq \gamma_{1} \\
\left.\frac{1}{\eta_{0}}+\frac{\beta_{2}}{\eta_{2}}\left(\frac{t-J_{\mathcal{N}_{t}}+\sum_{k=N_{\gamma_{1}}+1}^{N_{t}}\left[\prod_{z=k}\right.}{\eta_{2}}\right)^{N_{t}}\left(1-E_{z}\right)\right] x_{k} \\
\eta_{2}-1 & \text { if } t>\gamma_{1}
\end{array}\right.
$$

Being given that the virtual age just after the $k^{\text {th }}$ maintenance, noted $a_{k}$, is equal to

$$
\sum_{z=\mathcal{N}_{\gamma_{1}+1}}^{k}\left[\Pi_{h=z}^{k}\left(1-E_{h}\right)\right] x_{h} \text { for all } k \geq \mathcal{N}_{\gamma_{1}}+1
$$

where the variable $X_{h}$ indicate the $h^{\text {th }}$ duration of the between-failures.

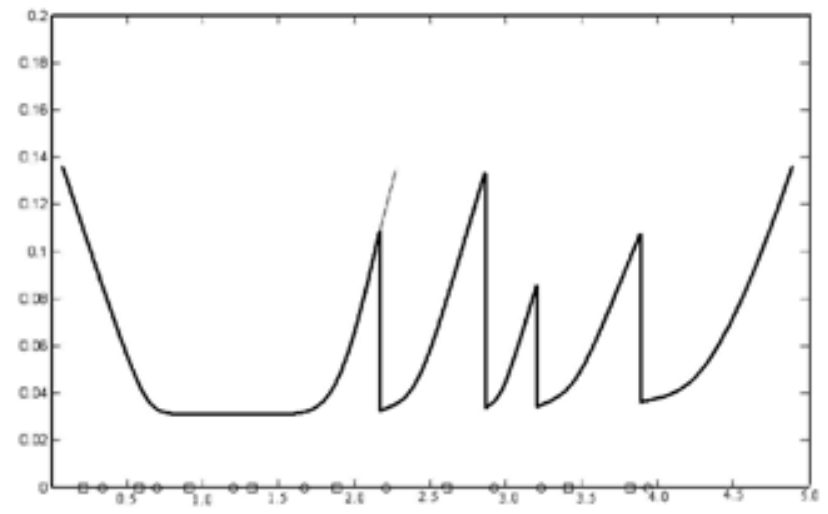

Figure 1. Reformulation of the Brown-Proschan model intensity

The figure 1 translated the trajectory of this intensity for an unspecified value of $p$ between 0 and 1 . In this figure, the instants of perfect and minimal maintenances are represented on the $\mathrm{x}$-axis respectively by circles and squares. 
Concerning the evaluation of the maintenance efficiency, we return to the same properties presented in Brown and Proschan (1983). The reformulation of the Block, Borges and Savits model is also a generalization of the preceding form of the Brown-Proschan model. Indeed, the probability that the CM is perfect depends to the instant to which is carried out. In this case, the failure intensity is equivalent to that of the Brown-Proschan model, with that the law of $E_{k}$ is related to parameters $p\left(\mathcal{T}_{k}\right)$.

\section{Characteristics of the failures process}

The model Brown-Proschan (BP) is a particular case of the Kijima model (1989), making the two part of the whole models of repair. A generalization of such a model arises in Last and Szekli (1998) shows that the failures process, under certain conditions, tends to be stabilized. For our BP reformulated model, and in the degradation phase (obviously, the failure intensity is monotonous), the convergence property in law of the virtual age after maintenance (or effective age) and the waiting durations between two failures, is checked. This fact, the virtual age just after the $k^{\text {th }}$ maintenance, noted $a_{k}$ have a distribution function of the form:

$$
\mathcal{F}_{a_{k}}(t)= \begin{cases}1-\exp \left\{-\frac{1}{\eta_{0}} t\right\} \times \exp \left\{-\left(\frac{t}{\eta_{1}}\right)^{\beta_{1}}+\left(\frac{\gamma_{0}}{\eta_{1}}\right)^{\beta_{1}-1} t\right\} & \text { if } 0<t<\gamma_{0} \\ 1-\exp \left\{-\frac{1}{\eta_{0}} t\right\} & \text { if } \gamma_{0} \leq t \leq \gamma_{1} \\ 1-(1-p) \exp \{-\Lambda(t)\}\left[\sum_{z=N_{\gamma_{1}+1}+1}^{k-1}(1-p)^{z} \frac{\Lambda^{z}(t)}{z !}\right] & \text { if } t>\gamma_{1}\end{cases}
$$

During the degradation period, and for a value of $p$ strictly higher than zero, the random variables continuation of the ages $\left\{a_{k}\right\}_{k \geq \mathcal{N}_{\gamma_{1}}+1}$ converge in law towards a random variable a. Brown and Proschan (1983) proved that this variable follows a law having the failure rate $p \lambda(t)$, and $\mathcal{F}_{a}(t)=1-(1-p) \exp \{-\Lambda(t)\}$ as function of distribution. It is the same, as constantly, the virtual age is equal to the time passed since the last perfect maintenance. In Last and Szekli (1998), the authors showed the convergence of the continuation of the virtual ages expectation, and of the expectation of the between-failures durations. Within the framework of our BP reformulated model, and thanks to the convergence property of Brown-Proschan, we can obviously calculate these expectations and prove their tendency towards a finished and continuous limit. 
The reformulation of the BP model using the failure intensity with bathtub form, characterize the failures process by the between-failures durations, for $k \geq \mathcal{N}_{\gamma_{1}}+1$, having the survival function as:

$$
\begin{aligned}
& S_{x_{k}}(x)=(1-p) \int_{\gamma_{1}}^{+\infty} \lambda(u) \exp \{-\Lambda(t+u)\} \\
& \quad\left[(1-p)^{k-2} \frac{\Lambda^{k-2}(u)}{(k-2) !}+p \sum_{z=N_{\gamma_{1}}}^{k-3}(1-p)^{z} \frac{\Lambda^{z}(u)}{z !}\right] d u+p \exp \{-\Lambda(x)\}
\end{aligned}
$$

And if moreover $p$ is strictly positive, and that $\exp \{-\Lambda(x)\}=o(1)$, then the survival function of the between-failures durations, for the values of $k \geq \mathcal{N}_{\gamma_{1}}+2$ converge in law towards the random variable $X$ with survival function:

$$
\mathcal{S}_{x}(x)=p \int_{\gamma_{1}}^{+\infty} \lambda(x+u) \exp \{-\Lambda(x+u)+(1-p) \Lambda(u)\} d u
$$

Thus, we deduce from these results, that the between-failures durations converge in law towards the random variable $X$ of which the survival function as:

$$
\delta_{X}(x)=p \exp \{-\Lambda(x)\}+p(1-p) \int_{\gamma_{1}}^{+\infty} \lambda(u) \exp \{-\Lambda(x+u)+(1-p) \Lambda(u)\} d u
$$

Consequently, by using an integration by parts, we can write:

$$
\begin{aligned}
& \delta_{x}(x)=\lim _{u \rightarrow+\infty} \exp \{-\Lambda(x+u)+(1-p) \Lambda(u)\} \\
& +p \int_{\gamma_{1}}^{+\infty} \lambda(x+u) \exp \{-\Lambda(x+u)+(1-p) \Lambda(u)\} d u
\end{aligned}
$$

And considering that $\Lambda$ is an increasing function, then:

$$
\exp \{-\Lambda(x+u)+(1-p) \Lambda(u)\} \leq \exp \{-p \Lambda(u)\}=o(1)
$$

Under these conditions, for our reformulation of the BP model by an intensity with bathtub form, if it exists an $\varepsilon>0$ so that: $x^{1+\varepsilon} \exp \{-\Lambda(x)\}=o(1)$ then the expectation of the average waiting duration of the $(k+1)^{t h}$ failure, with $k \geq \mathcal{N}_{\gamma_{1}}+1$ is expressed by the following relation:

$$
\mathbb{E}\left[x_{k}\right]=\int_{\gamma_{1}}^{+\infty} \exp \{-\Lambda(t)\}\left[(1-p)^{k-1} \frac{\Lambda^{k-1}(t)}{(k-1) !}+p \sum_{z=N_{\gamma_{1}}}^{k-2}(1-p)^{z} \frac{\Lambda^{z}(t)}{z !}\right] d t
$$




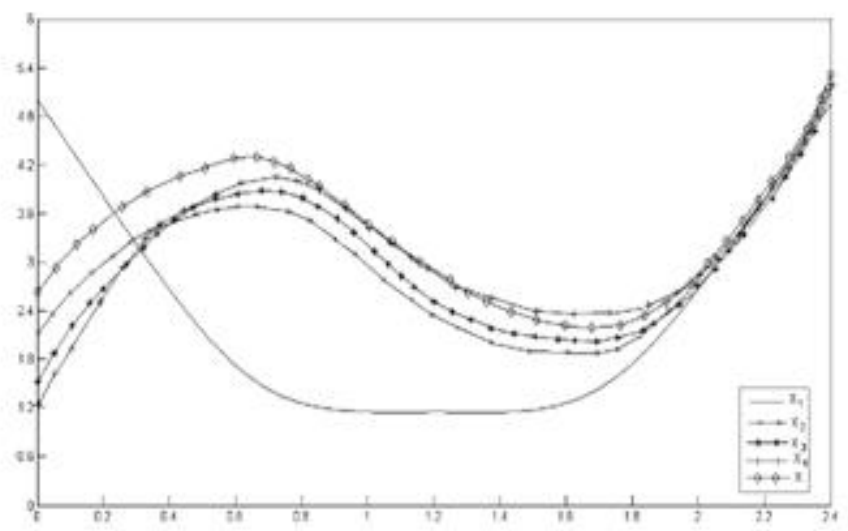

Figure 2. Random rate of the consecutive between-failures durations of the BP reformulated model

It appears that the most significant result of this section is, in the absence of any ambiguity, the relation (3) which offers the marginal laws of the consecutive between-failures durations. The figure 2 represents the simulated random rates of these between-failures durations of which the initial failure intensity in bathtub form

(defined by the parameters $\eta_{0}=26, \eta_{1}=\eta_{2}=200, \beta_{1}=0.5, \beta_{2}=3, \gamma_{0}=0.7, \gamma_{1}=1.6$ ) and a maintenance efficiency equalizes to 0.3 .

We notice that after the maintenance action, the random rate of the next betweenfailures duration is represented by a concave trajectory during the improvement period, and convex during the degradation period. The growth in $k$ of the first values of this random rate is due to the fact that the effect of this maintenance action on the system is unknown. In other word, just after a maintenance action, the system tends moreover to weaken because what we don't know if the maintenance be effective or not. After being maintained, if the system survives long enough, the random rate takes the values almost identical to the initial intensity (it's extremely probable that this last maintenance is perfect).

The chart of the asymptotic random rate of the between-failures durations (simulated with the same parameters values of the initial failure intensity of Fig.2) (Fig.3) illustrate the maintenance effect. It's obviously the form of the random rate associated to the variable $X$, already defined in the preceding paragraph, for various values of the efficiency parameter $p$. 


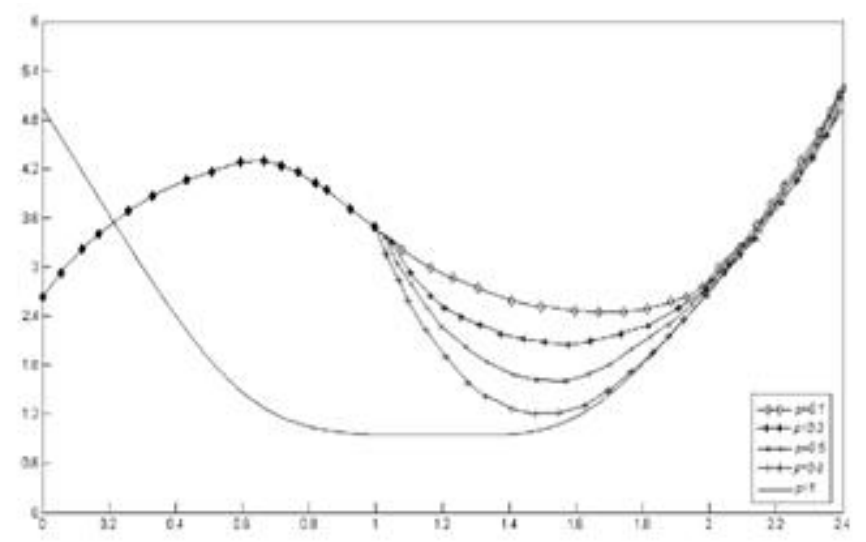

Figure 3. Asymptotic random rate of the between-failures durations according to various values of $p$

We see that during all the period before the beginning of the degradation phase, the form of the random rate keeps always the same pace whatever is the parameter value $p$. In fact the maintenanace effect is, by hypothesis, As Bad As Old $(A B A O)$, and the maintenance action is carried out just to take again the system operation). The maintenance effect, through the degradation period, is to increase the first values of the random rate compared to that of the initial intensity. This increase is even higher than the parameter $p$ is weak. So, more $p$ is weak and more the maintenance is extremely probable that it's ABAO.

\section{Estimate of the maintenance efficiency}

Under the assumption that the maintenance effects are known (the maintenance is As Good As New (AGAN) or ABAO, and $E_{k}$ are observed). Thus, the writing of the likelihood function is possible by using the equation (6). Therefore, we estimate the parameters of our model, such as the efficiency parameter $p$, and the parameters of the failure intensity $\lambda$. It's noticed that the estimator of $p$ is logically the percentage of the perfect maintenance actions among all actions carried out. In the case of failure intensity of the Power-Law-Process type, Whitaker and Samagniego (1989) studied the identifiability problem of the parameter $p$ for a waiting duration of the first failure according to the exponential law. And so the between-failures durations law is independent of the parameter $p$. Considering that the maintenance actions are useless when the system is neither in improvement state nor in degradation state, the models of assumptions AGAN and ABAO are the same ones.

$$
\mathcal{L}\left(t_{1}, \ldots, t_{n}\right)=\left[\prod_{i=1}^{n} \lambda_{t_{i}}\right] \exp \left\{-\sum_{i=1}^{n} \int_{t_{i-1}}^{t_{i}} \lambda_{s} d s\right\}
$$


If on the contrary, this first between-failure duration is supposed of nonexponential law, the identifiability problem does not arise and it's possible then to estimate in prior the parameter $p$. In the same way, we estimate the other parameters of the model, without having the observed values of the external variables $E$. Several alternatives of estimation, always for the simple failure intensity, namely the idea to use the Expectation Maximization (EM) algorithm, gotten by Lim (1998). In their article Lim, Lu and Park (1998) presented another method, based on the bayesian analysis, and which is trying to give to $p$ the prior law of the beta type. Another approach was proposed by Langseth and Lindqvist (2003), acted to calculate the model characterization without utilizing the external variables values $E$.

In practice, and without maintenance, the systems are considered either in improvement or in degradation states (in view of the service life phase is practically short). Then, the initial intensity cannot coincide with an exponential law and the identifiability problem doesn't presented. Indeed, this is logical insofar as the exponential law is in mental blank. Nevertheless, if the first between-failure duration is supposed of non-exponential law, the maintenance efficiency parameter is identifiable.

We interest thereafter, in the estimation of the parameters for our BP reformulated model by an intensity with bathtub form. The failures process depends on the external variables continuation of which we don't know their values. Subsequently, and even if the failures process is influenced by the external variables $E$, this can be in any event, also considered with a self-excited punctual process. The two following relations express the relationship between the failure intensities.

$$
\begin{gathered}
\lambda_{t}(\mathcal{N}, E)=\lim _{d t \rightarrow 0} \frac{1}{d t} \operatorname{Pr}\left(\mathcal{N}_{t+d t}-\mathcal{N}_{t}=1 / \mathcal{N}_{t}, \mathcal{T}_{N_{t}}, E_{N_{t}}\right) \\
\quad \text { and } \\
\lambda_{t}(\mathcal{N})=\lim _{d t \rightarrow 0} \frac{1}{d t} \operatorname{Pr}\left(\mathcal{N}_{t+d t}-\mathcal{N}_{t}=1 / \mathcal{N}_{t}, \mathcal{T}_{N_{t}}\right)
\end{gathered}
$$

We find in Andersen, Borgan and Gill (1993) the innovation theorem, allowing to note the failure intensity quite simply by $\lambda_{t}=\lambda_{t}(\mathcal{N})$. As the failure process is considered as well as a self-excited punctual process. We can apply within the parametric approach framework various procedures of estimate. This process is characterized by a clean failure intensity which is calculated in an iterative way according to the intensity values and cumulative failure intensity at the preceding maintenance instants. This function is expressed by the following equation. 


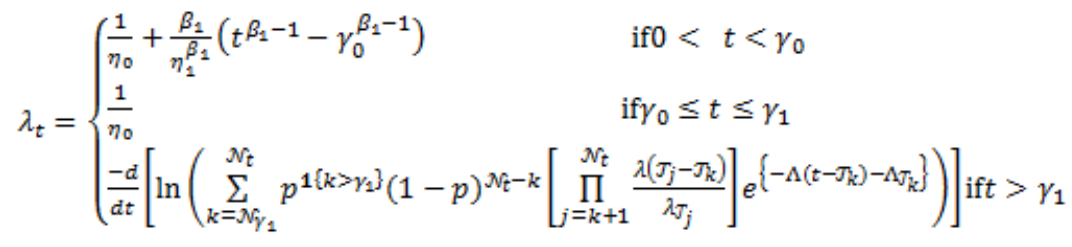

Obviously, through the degradation phase, this property formalizing the model BP. What is translated by the third restriction of the foregoing equation . For this fact, we condition the calculation compared to the instant of the last perfect maintenance action. Consequently, we note $E_{z, k}$, for $k \leq z$ and $k \geq$ the next event: the $k^{\text {th }}$ maintenance is AGAN and the following ones, until the $z^{\text {th }}$ maintenance, are ABAO:

$$
\begin{aligned}
E_{z, k}= & \left\{\left\{E_{k}=1\right\},\left\{E_{j}=0\right\}_{k<j \leq z}\right\}, \text { and for all } t \geq 0, \\
& \operatorname{Pr}\left(X_{n+1} \geq x / \mathcal{T}_{n}\right)=\exp \left\{-\int_{\mathcal{J}_{n}}^{J_{n}+x} \lambda_{u} d u\right\}
\end{aligned}
$$

When the law of the failure instants is influenced by the maintenances process, $M$, and by the external variables, $E$, the failure intensity remains insufficient to characterize perfectly the failures process. For this fact, and using the formula of the probability law of next between-failures time, data by the equation (7), and of the innovation theorem of Andersen, Borgan and Gill (1993) it's possible to write:

$$
\operatorname{Pr}\left(X_{n+1} \geq x / \mathcal{T}_{n}\right)=\exp \left\{-\int_{\mathcal{J}_{n}}^{\mathcal{J}_{n}+x} \mathbb{E}\left[\lambda_{u}^{\mathcal{N}}(\mathcal{N}, \mathcal{M}, E) / \mathcal{N}_{u}=n, \mathcal{T}_{n}\right] d u\right\}
$$

We can deduce from this last relation the law of between-failures times, knowing the history of the failures process. In our reformulation case of the BP model, and through the degradation period, the failures process checks:

$$
\operatorname{Pr}\left(X_{n+1} \geq x / \mathcal{T}_{n}\right)=\sum_{k=N_{\gamma_{1}}}^{n} p^{1\left\{k>\gamma_{1}\right\}}(1-p)^{n-k}\left[\prod_{j=k+1}^{n} \frac{\lambda\left(J_{j}-\mathcal{J}_{k}\right)}{\lambda J_{j}}\right] e^{\left\{-\Lambda\left(x+J_{n}-J_{k}\right)+\Lambda \tau_{n}-\Lambda \tau_{k}\right\}}
$$

Thus, during all its life, the system is characterized by a failures process, of which a law of the between-failures times is given by: 


$$
\begin{aligned}
& \operatorname{Pr}\left(X_{n+1} \geq x / \mathcal{T}_{n}\right)= \\
& \left\{\begin{array}{lc}
\exp \left\{-\frac{1}{\eta_{0}} x-\left(\frac{J_{n}+x}{\eta_{1}}\right)^{\beta_{1}}+\left(\frac{J_{n}}{\eta_{1}}\right)^{\beta_{1}}+\left(\frac{\gamma_{0}}{\eta_{1}}\right)^{\beta_{1}-1} x\right\} & \text { if } n<\mathcal{N}_{\gamma_{0}} \\
\exp \left\{-\frac{1}{\eta_{0}} x\right\} & \text { if } \mathcal{N}_{\gamma_{0}}<n<\mathcal{N}_{\gamma_{1}} \\
\sum_{k=N_{\gamma_{1}}}^{n} p^{1\left\{k>\gamma_{1}\right\}}(1-p)^{n-k}\left[\prod_{j=k+1}^{n} \frac{\lambda\left(J_{j}-\mathcal{J}_{k}\right)}{\lambda J_{j}}\right] e^{\left\{-\Lambda\left(x+J_{n}-\mathcal{J}_{k}\right)+\Lambda J_{n}-\Lambda J_{k}\right\}_{1}} \text { if } n>\mathcal{N}_{\gamma_{1}}
\end{array}\right.
\end{aligned}
$$

We hold to distinguish between this property and that from the survival function. The Interpretations of the two properties are considerably distinct. The property corresponding to the survival function transmitted the marginal law of the consecutive between-failures durations. What allows us to well understand the evolution of the failures process. Whereas, the property of the preceding equation presents the conditional law of the consecutive between-failures durations. These two laws entirely characterize the failures process. Remain the problem of complexity which meets us in their studies.

We find in the figure 4 our reformulation with bathtub form of the self-excited failure intensity (in full feature), $\lambda_{t}$ and the failure intensity relating to the external process $\lambda_{t}(\mathcal{N}, E)$. The AGAN maintenance actions are indicated on the instants axis by circles, and the ABAO maintenance actions by squares. Subsequent to a maintenance action, the self-excited failure intensity proves to be the form of the initial intensity (in bathtub form). When the duration passed since the preceding maintenance action is sufficiently long, the self-excited failure intensity is equal to the initial intensity at the instant $t-\mathcal{T}_{\mathcal{N}_{t}}$. In consequence of a maintenance action, the pace of the self-excited failure intensity is dependent on the history of the failures process. Indeed, if the previous between-failures duration is sufficiently high then the length of the improvement period is less significant.

By using the self-excited failure intensity defined, we can deduce the likelihood function associated to the observation of the maintenance instants. Under these conditions, our BP reformulated model allows to withdraw the likelihood function associated to only one observation of the failures process. By using the equation (6), this function is given by:

$$
\mathcal{L}_{t_{n}}\left(\theta ; t_{1}, \ldots, t_{n}\right)=\left[\prod_{k=1}^{n} \lambda_{J_{k}}\right] \exp \left\{-\sum_{k=1}^{n} \int_{t_{k-1}}^{t_{k}} \lambda_{s} d s\right\}=\left[\prod_{k=1}^{n} \lambda_{J_{k}}\right] \exp \left\{-\Lambda_{t_{n}}\right\}
$$

This last function is equivalent to the likelihood function developed in the work of Doyen (2010). And that the restriction of this likelihood function over the two improvement and service life periods is equal to: 


$$
\begin{gathered}
\mathcal{L}_{t_{n}\left\{n \leq \mathcal{N}_{\gamma_{1}}\right\}}\left(\theta ; t_{1}, \ldots, t_{n}\right)=\left[\prod_{k=1}^{i}\left(\frac{1}{\eta_{0}}+\frac{\beta_{1}}{\eta_{1}^{\beta_{1}}}\left(t_{k}^{\beta_{1}-1}-\gamma_{0}^{\beta_{1}-1}\right)\right)\right] \\
\times\left(\frac{1}{\eta_{0}}\right)^{n-i} \times \exp \left\{-\left(\frac{\gamma_{0}}{\eta_{1}}\right)^{\beta_{1}}-\frac{1}{\eta_{0}} t_{n}\right\}
\end{gathered}
$$

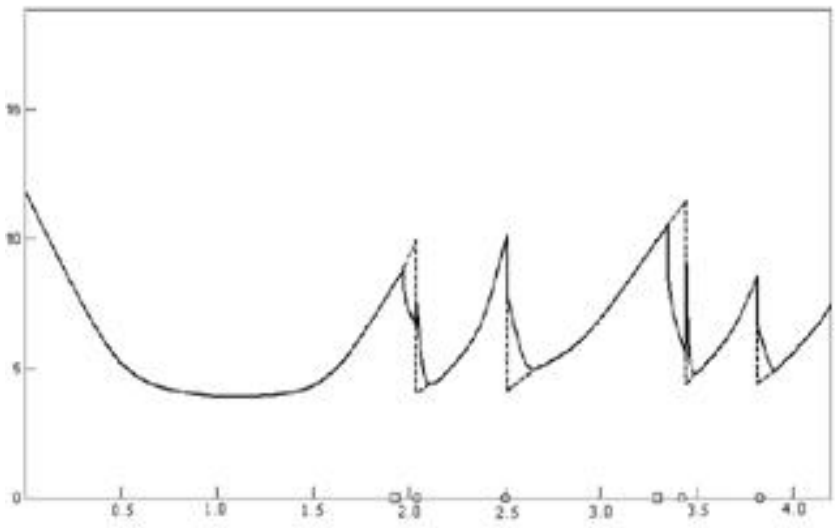

Figure 4. Reformulation in the bath-tub form of the self-excited and relative intensities of the BP model

Whereas through the degradation period, the restriction of this likelihood function is expressed as:

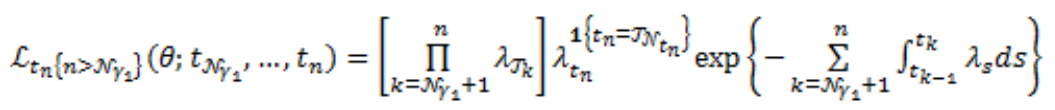

Then the restriction likelihood function is written:

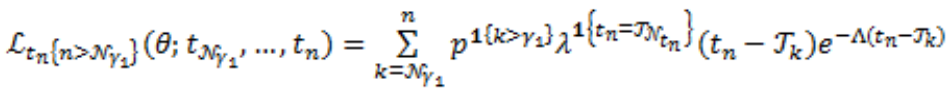

$$
\begin{aligned}
& (1-p)^{n-k}\left[\prod_{j=k+1}^{n} \lambda\left(\mathcal{T}_{j}-\mathcal{T}_{k}\right)\right]\left[\prod_{j=N_{\gamma_{1}+1}}^{k} \lambda \lambda_{J_{j}}\right] e^{-\Lambda \tau_{k}} \\
& =\lambda^{1\left\{t_{n}=\mathcal{J}_{N_{t_{n}}}\right\}}\left(t_{n}\right) e^{-\Lambda\left(t_{n}\right)}(1-p)^{n}\left[\prod_{j=\mathcal{N}_{Y_{1}+1}}^{n} \lambda\left(t_{n}-\mathcal{T}_{j}\right)\right]+\sum_{k=\mathcal{N}_{Y_{1}+1}}^{n} p \lambda^{1\left\{t_{n}=\mathcal{J N}_{t_{n_{n}}}\right\}} \\
& \left(t_{n}-\mathcal{T}_{k}\right) e^{-\Lambda\left(t_{n}-\mathcal{T}_{k}\right)}(1-p)^{n-j}\left[\prod_{j=k+1}^{n} \lambda\left(\mathcal{T}_{j}-\mathcal{T}_{k}\right)\right]\left[\prod_{j=\aleph_{\gamma_{1}+1}}^{k} \lambda \tau_{J_{j}}\right] e^{-\Lambda T_{k}}
\end{aligned}
$$

And owing to the fact that:

$$
\mathcal{L}_{\tau_{k}}\left(\theta ; t_{\mathcal{N}_{\gamma_{1}}}, \ldots, t_{k}\right)=\left[\prod_{j=1}^{k} \lambda_{J_{j}}\right] \exp \left\{-\Lambda_{\tau_{k}}\right\}
$$

Therefore, while associating to the $n^{\text {th }}$ observation, such that $n>\mathcal{N}_{\gamma_{1}}$, we obtain: 


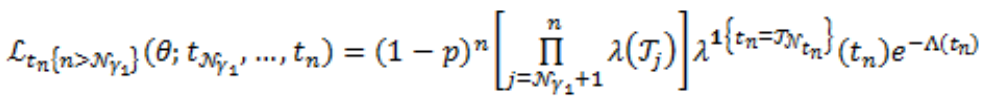

$$
\begin{aligned}
& +p\left[\sum_{k=N_{Y_{1}+1}}^{n}(1-p)^{n-k}\left(\prod_{j=k+1}^{n} \lambda\left(\mathcal{T}_{j}-\mathcal{T}_{k}\right)\right) \lambda^{1\left\{t_{n}=\mathcal{J}_{N_{n}}\right\}}\left(t_{n}-\mathcal{T}_{k}\right) e^{-\Lambda\left(t_{n}-\mathcal{T}_{k}\right)} \mathcal{L}_{\mathcal{J}_{k}}(\theta)\right]
\end{aligned}
$$

Finally, the likelihood function of our reformulation of the BP model is overall definite by:

$$
\mathcal{L}_{t_{n}}\left(\theta ; t_{1}, \ldots, t_{n}\right)=\mathcal{L}_{t_{n}\left\{n \leq N_{\gamma_{1}}\right\}}\left(\theta ; t_{1}, \ldots, t_{n}\right) \times \mathcal{L}_{t_{n}\left\{n>N_{\gamma_{1}}\right\}}\left(\theta ; t_{\left.N_{\gamma_{1}}, \ldots, t_{n}\right)}\right.
$$

It appears that in our study, the fact of removing the logarithm of the likelihood function doesn't simplify calculations. Thereafter, we attach to the direct calculation of the first partial derivative of likelihood in $p$. This calculation is resulted in the following function:

$$
\begin{aligned}
& \frac{\partial}{\partial p} \mathcal{L}_{t_{n}}\left(\theta ; t_{1}, \ldots, t_{n}\right)= \\
& {\left[\prod_{k=1}^{i}\left(\frac{1}{\eta_{0}}+\frac{\beta_{1}}{\eta_{1}^{\beta_{1}}}\left(t_{k}^{\beta_{1}-1}-\gamma_{0}^{\beta_{1}-1}\right)\right)\right]\left(\frac{1}{\eta_{0}}\right)^{n-i} e^{-\left(\frac{\gamma_{0}}{\eta_{1}}\right)^{\beta_{1}}-\frac{1}{\eta_{0}} t_{n}} \times\left\{-n(1-p)^{n-1}\right.} \\
& {\left[\prod_{j=N_{V_{1}+1}}^{n} \lambda\left(\mathcal{T}_{j}\right)\right] \lambda^{1\left\{t_{n}=\mathcal{J}_{N_{N_{n}}}\right\}}\left(t_{n}\right) e^{-\Lambda\left(t_{n}\right)}+\sum_{k=N_{V_{1}+1}}^{n}(1-p)^{n-k-1}\left(\prod_{j=k+1}^{n} \lambda\left(\mathcal{T}_{j}-\mathcal{T}_{k}\right)\right)} \\
& \left.\lambda^{1\left\{t_{n}=\mathcal{J}_{N_{T_{n}}}\right\}}\left(t_{n}-\mathcal{T}_{k}\right) e^{-\Lambda\left(t_{n}-\mathcal{J}_{k}\right)}\left[(1-(n-k+1) p) \mathcal{L}_{\mathcal{J}_{k}}(\theta)+p(1-p) \frac{\partial}{\partial p} \mathcal{L}_{\mathcal{J}_{k}}(\theta)\right]\right\}
\end{aligned}
$$

Given that the calculation of the partial derivative of likelihood in the parameters of the initial intensity is much more complex, we can then use their estimators without the maintenance process. Indeed, the estimate is carried out being given only the failures process and the maintenance actions are supposed as minimal. Moreover, the two estimate procedures presented (the direct maximum likelihood and the EM algorithm) get in their globally the best estimators, especially that of the EM algorithm.

\section{Conclusion}

In this study, we gave new results on our new reformulation of the Brown-Proschan model. Doyen (2010) proved that this model corresponds to systems for which the maintenance efficiency makes it possible to contain or to stabilize degradation. That enabled us to introduce the innovation theorem which makes it possible to treat our general model of maintenance efficiency including the hidden external variables, in a way similar to a self-excited punctual process. 
In the simulation stage, it's noticed that the service life period was not taken into account, in the direction where the system state is stabilized during this period. Moreover, in practice the service life period is, in general, short compared to the total life period of the reparable systems.

We can then, for this general model, to calculate a failure intensity known as clean and a clean likelihood function. Within the framework of the BP model, this failure intensity and this clean likelihood are complex and must be calculated recursively. Moreover, in spite of the complexity of the clean likelihood function, we showed that it was possible to calculate its partial derivative and thus to maximize it by numerical methods.

By knowing the clean likelihood function, other methods of estimate can be used. Like a method of Newton or via groping by calculating all the clean likelihood values on a grid.

\section{References}

Andersen, P.K., Borgan, O., \& Gill, R.D. (1993). Statistical models based on counting processes. Springer Series in Statistics, Springer-Verlag.

Baxter, L., Kijima, M., \& Tortorella, M. (1996). A point process model for the reliability of a maintained system subject to general repair. Communications in Statistics, 12, 37-65.

Block, H., Borges, W.S., \& Savits, T.H. (1985). Age-dependent minimal repair. Journal of Applied Probability, 22, 370-385. http://dx.doi.org/10.2307/3213780

Brown, M., \& Proschan, F. (1983). Imperfect Repair. Journal of Applied Probability, 20, 851-859. http://dx.doi.org/10.2307/3213596

Dijoux, Y. (2009). A virtual age model based on a bathtub shaped initial intensity. Reliability Engineering and System Safety, 94, 982-989. http://dx.doi.org/10.1016/j.ress.2008.11.004

Dorado, C., Hollander M., \& Sethuraman J. (1997). Nonparametric estimation for a general repair model. The Annals of Statistics 25(3), 1140-1160. http://dx.doi.org/10.1214/aos/1069362741

Doyen, L. (2004). Estimation of Maintenance efficiency in imperfect repair models, mathematical methods in reliability. Santa Fe, USA. 
Doyen, L. (2010). Asymptotic properties of imperfect repair models and estimation of repair efficiency. Naval Research Logistics, 57(3), 296-307.

Finkelstein, M.S. (2008). Failure rate modelling for risk and reliability. Springer.

Jiang, R., Ji, P., \& Xiao, X. (2003). Aging property of unimodel failure rate models. Reliability Engineering and System Safety, 79(1), 113-116. http://dx.doi.org/10.1016/S0951-8320(02)00175-8

Kijima, M. (1989). Some results for repairable systems with general repair. Journal of Applied Probability, 26, 89-102. http://dx.doi.org/10.2307/3214319

Langseth, H., \& Lindqvist, B.H. (2003). A maintenance model for components exposed to several failure mechanisms and imperfect repair, quality, reliability and engineering statistics. World Scientific Publishing.

Last, G., \& Szekli, R. (1998). Asymptotic and monotonicity properties of some repairable systems. Advances in Applied Probability, 30, 1089-1110. http://dx.doi.org/10.1239/aap/1035228209

Lim, T.J. (1998). Estimating system reliability with fully masked data under BrownProschan imperfect repair model. Reliability Engineering and System Safety 59, 277-289. http://dx.doi.org/10.1016/S0951-8320(97)00085-9

Lim, T.J., Lu, K.L., \& Park, D.H. (1998). Bayesian imperfect repair model. Communications in Statistics-Theory and Methods, 27(4), 965-984. http://dx.doi.org/10.1080/03610929808832138

Wang, H. (2002). A survey of maintenance policies of deteriorating systems. European Journal of Operational Research, 139(3), 469-489. http://dx.doi.org/10.1016/S0377-2217(01)00197-7

Whitaker, L.R., \& Samaniego, F.J. (1989). Estimating the reliability of systems subject to imperfect repair. Journal of the American Statistical Association, 84, 301-309. http://dx.doi.org/10.2307/2289878

Journal of Industrial Engineering and Management, 2012 (www.jiem.org)

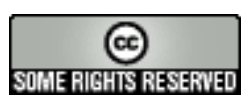

\footnotetext{
Article's contents are provided on a Attribution-Non Commercial 3.0 Creative commons license. Readers are allowed to copy, distribute and communicate article's contents, provided the author's and Journal of Industrial Engineering and Management's names are included. It must not be used for commercial purposes. To see the complete license contents, please visit http://creativecommons.org/licenses/by-nc/3.0/.
} 